\title{
Comparison of Low Dose versus High Dose of Oxytocin for Initiating Uterine Contraction During Cesarean Delivery: A Randomized, Controlled, Non-Inferiority Trial
}

This article was published in the following Dove Press journal:

International Journal of Women's Health

\author{
Monsicha Somjit' \\ Jaruta Surojananon' \\ Kiattisak Kongwattanakul (D) ${ }^{2}$ \\ Cattleya Kasemsiri (iD) \\ Monthira Sirisom \\ Khuantipa Prawannoa' \\ Kaewjai Thepsuthammarat $\mathbb{D}^{3}$ \\ Ratana Komwilaisak (D) ${ }^{2}$ \\ 'Department of Anesthesiology, Faculty \\ of Medicine, Khon Kaen University, Khon \\ Kaen, Thailand; ${ }^{2}$ Department of \\ Obstetrics and Gynecology, Faculty of \\ Medicine, Khon Kaen University, Khon \\ Kaen, Thailand; ${ }^{3}$ Clinical Epidemiology \\ Unit, Faculty of Medicine, Khon Kaen \\ University, Khon Kaen, Thailand
}

Background: Oxytocin is used for initiating uterine contraction and preventing postpartum hemorrhage during caesarean delivery. Using a lower dosage of oxytocin may lower the risk of adverse effects while still being effective in stimulating initial uterine contraction. We aimed to compare the effectiveness and side effects of the standard 10 IU bolus of oxytocin with those of a 5 IU bolus during caesarean delivery.

Patients and Methods: We enrolled women in a randomized, double-blind, study comparing intravenous injections of high-dose (10 IU) and low-dose (5 IU) oxytocin administered after clamping of the umbilical cord. The primary outcome was adequate uterine contraction within the first 3 mins after administration. Secondary outcomes included uterine tone, use of additional uterotonic agents, additional obstetrics procedures, and oxytocin-related adverse events.

Results: A total of 155 women underwent randomization, with 78 in the low-dose group and 77 in the high-dose group. The proportion of women with adequate uterine contraction during the first 3 mins was $84.6 \%$ in the low-dose group and $77.9 \%$ in the high-dose group (relative risk, $1.09 ; 95 \%$ CI, 0.93 to 1.26 ). Methylergonovine maleate was used in $14.1 \%$ of cases in the low-dose group and $36.4 \%$ in the high-dose group (relative risk, $0.40 ; 95 \% \mathrm{CI}, 0.22$ to 0.73 ). The necessity for additional obstetric procedures, estimated blood loss $>500 \mathrm{~mL}$, neonatal outcomes, and oxytocinrelated adverse effects did not differ significantly between the two groups.

Conclusion: The 5 IU bolus of oxytocin was noninferior to the standard 10 IU bolus of oxytocin for initiating adequate uterine contraction, required fewer additional uterotonic agents, and led to fewer oxytocin-related adverse events.

Keywords: oxytocin, cesarean section, uterine contraction

\section{Introduction}

Postpartum hemorrhage (PPH) remains a leading direct cause of maternal death worldwide, representing $27.1 \%$ of maternal deaths ${ }^{1}$ and significant maternal morbidity. ${ }^{2-5}$ The most common cause of PPH (in an estimated $70 \%$ of cases) is failure of adequate uterine contraction, or uterine atony. Cesarean delivery has also been shown to increase the risk of PPH. ${ }^{6}$ Importantly, 54-93\% of maternal deaths due to obstetric hemorrhage may be preventable. ${ }^{7,8}$

Oxytocin is used in obstetrics to promote uterine contraction and is the agent of choice in the prevention of postpartum uterine atony during caesarean delivery. The
Correspondence: Kiattisak Kongwattanakul

Department of Obstetrics and Gynecology, Faculty of Medicine, Khon Kaen University, Khon Kaen 40002

Thailand

Tel +66935949542

Fax +6643348359

Email Kiattisak@kku.ac.th
International Journal of Women's Health 2020:12 667-673

667

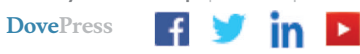

http://doi.org// 0.21 47/IJWH.S260073 
use of oxytocin has reduced maternal morbidity worldwide. While maternal mortality rates due to postpartum hemorrhage have decreased in the last four decades, PPH still accounts for more than $10 \%$ of pregnancy-related deaths. ${ }^{9}$ In addition, use of oxytocin is not without its own problems as it can cause significant maternal side effects, eg, nausea, vomiting, headache, flushing, water retention, hyponatremia, seizure, and coma. ${ }^{10}$

In 2012, the WHO published its "Recommendations for Prevention and Treatment of Postpartum Hemorrhage," in which it stated that all women giving birth should be offered a uterotonic during the third stage of labor for prevention of PPH, with Oxytocin (IM/IV 10 IU) being the uterotonic of choice. $^{9}$ The Royal College of Obstetricians and Gynecologists published guidelines on the "Prevention and Management Postpartum Hemorrhage," in which they recommended that oxytocin (5 IU by slow intravenous injection) be used to encourage contraction of the uterus and to decrease blood loss in women delivering by caesarean section. ${ }^{11}$ The French College of Gynaecologists and Obstetricians with the French Society of Anesthesiology and Intensive Care recommended an initial administration of 5-10 IU of IV or IM oxytocin injected slowly after caesarean delivery. $^{12}$

There is currently no standard protocol for the prevention of postpartum hemorrhage nor for the dosage of oxytocin to be used. We hypothesized that using a lower dose of oxytocin may be as effective as a higher dose in stimulating initial uterine contraction while leading to fewer side effects. We thus aimed to compare a standard 10 IU bolus of oxytocin with a 5 IU bolus in these regards.

\section{Patients and Methods}

\section{Setting and Participants}

We performed a randomized, double-blind, controlled, non-inferiority trial between November 1, 2018 and August 31, 2019 at Khon Kaen University's Srinagarind hospital, a tertiary hospital in Thailand, to compare the effects of different doses of oxytocin among women undergoing planned Cesarean delivery. This study was conducted and reported according to the CONSORT Statement. ${ }^{13}$ The trial registration number of the protocol for this study is TCTR20190415001. Pregnant women were not involved in the development of the study.

The eligibility criteria were singleton pregnancy, age 18-40 years, 37-41 completed weeks of gestational age, American Society of Anesthesiology (ASA) physical status class II, and scheduled cesarean delivery under spinal anesthesia. Women were excluded in whom spinal anesthesia had failed or was inadequate, who had undergone uterine surgery other than cesarean section, who had a high risk of uterine atony (macrosomia, chorioamnionitis, polyhydramnios, uterine mass) or postpartum hemorrhage (placenta previa or other placenta disorders, history of postpartum hemorrhage, coagulopathy, thrombocytopenia, or pre-eclampsia), or who had known allergies to oxytocin. Written informed consent was obtained from all eligible participants during their admission to the labor ward.

\section{Randomization and Interventions}

The randomization sequence was computer-generated by a biostatistician, and allocations were sealed in opaque envelopes to ensure concealment. Women were randomly assigned to receive a single intravenous bolus injection of oxytocin at a dose of either $5 \mathrm{IU}$ or $10 \mathrm{IU}$.

Data regarding baseline characteristics and anesthesia were recorded. Pre-operative blood pressure and heart rate were measured. All women received a preload of Ringer's lactate solution. Spinal anesthesia was induced using hyperbaric bupivacaine $(0.5 \%$ heavy Marcaine $10-15 \mathrm{mg}$ with Morphine $0.1-0.2 \mathrm{mg}$ ). Women were moved to a supine position with left uterine displacement. Surgery commenced when sensory block to pinprick was confirmed as anesthetic level T4-T6. Immediately after the birth of the baby (T0), patients received either a 5 IU or $10 \mathrm{IU}$ bolus of oxytocin over $15 \mathrm{~s}$, according to group assignment. Uterine tone, blood pressure, heart rate and adverse events were recorded at 1, 2, 3, 6, 9, and 15 mins. At 3 mins after delivery, all patients received $1000 \mathrm{~mL}$ of Ringer's lactate solution with 20 IU of oxytocin by intravenous drip (125 mL/hr; $2.5 \mathrm{IU} / \mathrm{hr})$.

We focused on uterine contraction and tone during the first 3 mins of administration and added oxytocin infusion after 3 mins because the plasma half-life of oxytocin is 3 to $5 \mathrm{mins}$ and myometrium response is almost immediate. ${ }^{14}$ Uterine contraction was assessed by a blinded obstetric surgeon as either adequate or inadequate, and uterine tone was assessed using a linear analogue scale (0-10). If the uterine contraction was deemed "inadequate" or additional uterotonic agents were required, they were administered following the rescue protocol established by the anesthesiologists involved in the study. The first-step rescue agents in the low-dose group were $5 \mathrm{IU}$ of intravenous oxytocin administered 
over $15 \mathrm{~s}$ and the followed steps agents in both groups were methylergonovine maleate (0.2 mg, intravenous), misoprostol (800 $\mu \mathrm{g}$, rectal suppository) and sulprostone (500 $\mu \mathrm{g}$, intravenous drip over 30 mins). Any other adverse events were managed at the discretion of the attending anesthesiologists.

\section{Outcome Measures}

The primary outcome was adequate uterine contraction within the first 3 mins after administration. The secondary outcomes included uterine tone, use of additional uterotonic agents, additional obstetrics procedures (uterine artery ligation, B-lynch procedure and hysterectomy), estimated blood loss (by calculating the blood volume of the suction machine and weighing swabs), preoperative and 2-day postoperative hematocrit, blood transfusion, and oxytocin-related adverse events (vomiting, flushing, tachycardia, heart rate $\geq 120$ beats/minutes, hypotension, mean arterial pressure decrease $\geq 20 \%$ from baseline, chest pain, and EKG change). Neonatal outcomes included birth asphyxia, defined as an Apgar score $<7$ at 5 mins after birth together with admission to a neonatal intensive care unit (NICU) and neonatal resuscitation.

\section{Statistical Analysis}

A non-inferiority design was chosen since the aim of the trial is to determine if $5 \mathrm{IU}$ oxytocin is non-inferior in efficacy to the standard intervention (oxytocin $10 \mathrm{IU}$ ). In order to demonstrate non-inferiority within a margin of $0.1 \%$, with a power of $80 \%$, and with a significance level of $5 \%$, a total of 160 women were required assuming an equal adequate uterine contraction of $93.5 \%$ with treatment. ${ }^{15}$

Data were analyzed on an intention-to-treat basis. Descriptive statistics were used to report the baseline characteristics of the participants. The risk ratios (RRs) and the respective $95 \%$ confidence intervals (CIs) were calculated to indicate the between-group differences. All analyses were carried out using STATA 10 (Stata Corporation, College Station, TX, USA). The deidentified data that support the findings of this study are available from the corresponding author, $[\mathrm{KK}]$, upon reasonable request.

\section{Results}

Of 389 women who were eligible for enrolment, 160 were included and randomly assigned to either the low-dose or high-dose oxytocin group. Outcomes were excluded of 5 participants, 2 in the low-dose group (1 leiomyoma and 1 fetal macrosomia) and 3 in the high-dose group (2 leiomyoma and 1 macrosomia), leaving 78 and 77 participants for analysis in low-dose and high-dose group, respectively. See Figure 1 for the flow diagram of the study.

Demographic of obstetric and anesthetic characteristics at baseline were similar in the two groups (Table 1). The primary outcome of adequate uterine contraction during the first 3 mins of oxytocin administration occurred in a total of

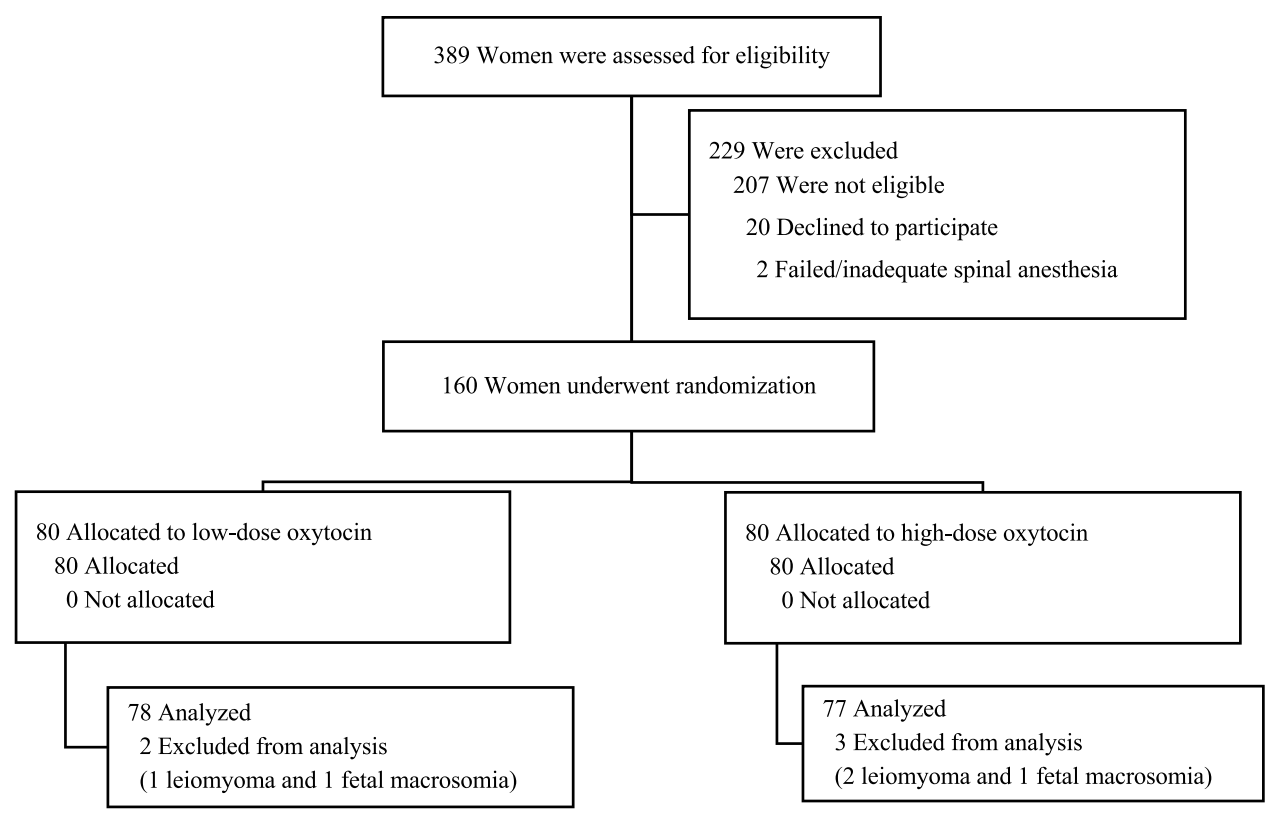

Figure I Consort flowchart of the study. 
Table I Baseline Characteristics

\begin{tabular}{|c|c|c|}
\hline Characteristics & $\begin{array}{l}\text { Low-Dose } \\
\text { Oxytocin } \\
(\mathrm{N}=78)\end{array}$ & $\begin{array}{l}\text { High-Dose } \\
\text { Oxytocin } \\
(\mathrm{N}=77)\end{array}$ \\
\hline $\begin{array}{l}\text { Mean age - years } \\
\geq 35\end{array}$ & $\begin{array}{l}30.13(4.77) \\
18(23.1)\end{array}$ & $\begin{array}{l}31.14(4.48) \\
17(22.1)\end{array}$ \\
\hline $\begin{array}{l}\text { Mean BMI - kg/m² } \\
\text { Nulliparous, } n(\%)\end{array}$ & $\begin{array}{l}28.49(3.84) \\
35(44.9)\end{array}$ & $\begin{array}{l}28.59(3.71) \\
30(39.0)\end{array}$ \\
\hline $\begin{array}{l}\text { Mean gestational age - week } \\
37-38^{+6} \\
39-41\end{array}$ & $\begin{array}{l}38.28(0.91) \\
54(69.2) \\
24(30.8)\end{array}$ & $\begin{array}{l}38.19(0.84) \\
57(74.0) \\
20(20.0)\end{array}$ \\
\hline In labor stage, n (\%) & $26(33.3)$ & $29(37.7)$ \\
\hline $\begin{array}{l}\text { Indication of cesarean } \\
\text { section, } \mathbf{n}(\%) \\
\text { Previous Cesarean section } \\
\text { Cephalopelvic disproportion } \\
\text { Fetal condition } \\
\text { Other }\end{array}$ & $\begin{array}{l}34(43.6) \\
25(32.1) \\
11(14.1) \\
8(10.3)\end{array}$ & $\begin{array}{l}44(57.1) \\
20(26) \\
7(9.1) \\
6(7.8)\end{array}$ \\
\hline $\begin{array}{l}\text { Anesthetic level, } \mathbf{n} \text { (\%) } \\
\text { T4 } \\
\text { T5 } \\
\text { T6 }\end{array}$ & $\begin{array}{l}42(54.6) \\
2(2.6) \\
32(41.6)\end{array}$ & $\begin{array}{l}51(66.2) \\
4(5.2) \\
22(28.6)\end{array}$ \\
\hline $\begin{array}{l}\text { Preoperative baseline } \\
\text { Mean arterial pressure }(\mathrm{mmHg}) \\
\text { Mean heart rate (beat/mins) } \\
\text { Intravenous fluid preload }(\mathrm{mL}) \\
\text { Hematocrit }(\%)\end{array}$ & $\begin{array}{l}81.83(10.88) \\
84.64(16.48) \\
932.05(265.67) \\
34.31(3.12)\end{array}$ & $\begin{array}{l}83.94(11.75) \\
87.64(16.95) \\
936.36(252.83) \\
35.06(3.89)\end{array}$ \\
\hline Birth weight - g & $3212.60(347.75)$ & $3188.44(341.27)$ \\
\hline
\end{tabular}

Notes: Data are presented as mean \pm standard deviation or number (percentage). Abbreviations: BMI, body mass index; GA, gestational age.

126 participants (81.3\%), 66 (84.6\%) in the low-dose oxytocin group and $60(77.9 \%)$ in the high-dose oxytocin group (relative risk, 1.09; 95\% confidence interval [CI], 0.93 to 1.26 ).

Secondary outcomes are shown in Table 2. Mean uterine tone at 3 mins, rated as poor, moderate, or strong contraction, did not differ significantly between the two groups (7.60, SD 1.05 in the low-dose group versus 7.64, SD 0.85 in the high-dose group, mean difference 0.01 ; $95 \% \mathrm{CI},-0.32,0.35)$. Significantly fewer women allocated to the low-dose oxytocin group required methylergonovine maleate ( 14.1 versus $36.4 \%$, relative risk, $0.40 ; 95 \% \mathrm{CI}$ 0.22 to 0.73 ). None of the participants required additional obstetrical procedures, received blood transfusion, or had estimated blood loss of more than $1000 \mathrm{~mL}$. There were no significant differences of the hematocrit drop more than $3 \%$ between the two groups. In terms of neonatal outcomes, there were fewer cases of neonatal resuscitation and NICU admission in the low-dose oxytocin group, and there were no cases of birth asphyxia in either group.

There were no differences at the $5 \%$ level of significance between groups in the rates of the oxytocin-related adverse effects (Table 3). Accordingly we need to find out the adverse effects related with initial dose of the oxytocin. Thus, we excluded patients from this analysis in whom adverse events that occurred due to other additional uterotonic agents, leaving 72 women in the low-dose oxytocin group and 70 women in the high-dose oxytocin group. Women allocated to the low-dose oxytocin group were less likely to experience both mild (flushing and tachycardia) and severe (hypotension, chest pain, and ECG change) adverse effects, but not to a statistically significant extent. The incidence of hypotension, in particular, was lower (but not significantly so) in the low-dose group (30.6\% versus $42.9 \%$, relative risk; $0.72,95 \%$ CI 0.46 to 1.11 ). No vomiting occurred in either group.

\section{Discussion}

In this double-blind, randomized trial, we found that the intravenous administration of $5 \mathrm{IU}$ of oxytocin was noninferior to that of $10 \mathrm{IU}$ for initiating uterine contraction during cesarean delivery and that fewer patients administered the former dosage required methylergonovine maleate. Secondary outcomes (uterine tone, additional obstetrics procedures, estimated blood loss, and oxytocinrelated adverse events) did not differ significantly between the two groups.

The present trial provides a large-scale non-inferiority evaluation of oxytocin at a lower dosage against the standard dosage. There have been three previous studies comparing oxytocin at different doses. Sarna et al compared four different doses of oxytocin to determine its minimal effective dose during elective cesarean section and found that there were no differences in the uterine tone in the four groups. ${ }^{16}$ The Butwick et al studied the lower doses of oxytocin (0.5-3 units) that can produce the adequate uterine tone as the 5 units oxytocin and the prevalence of hypotension was significantly higher after 5 units oxytocin (47\%). ${ }^{17}$ Derbel et al studied to compare the 5 IU versus 10 IU bolus during Cesarean section and there were no significant differences between the two groups regarding blood loss and type of side effects that were more common in the $10 \mathrm{IU}$. This finding consistent with the present results. ${ }^{18}$

We propose three possible explanations for our finding that $5 \mathrm{IU}$ of oxytocin was adequate for initiating uterine 
Table 2 Primary and Secondary Outcomes

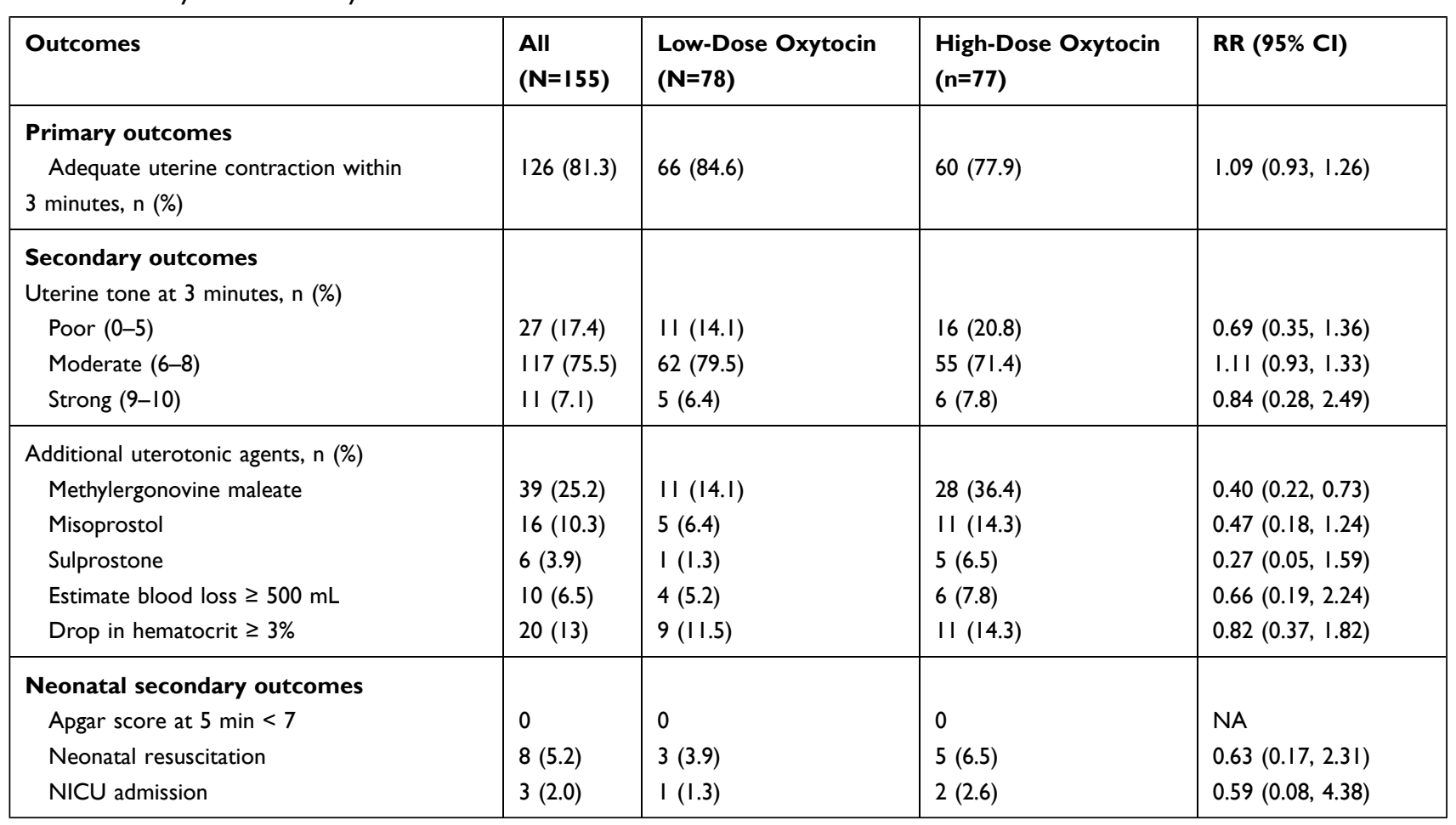

Notes: Data are presented as number (percentage). Low-dose oxytocin is the reference group.

Abbreviations: $\mathrm{RR}$, relative risk; $\mathrm{Cl}$, confidence interval.

Table 3 Oxytocin-Related Adverse Effects Outcomes

\begin{tabular}{|c|c|c|c|c|}
\hline Adverse Effects & All $(N=\mid 42)$ & $\begin{array}{l}\text { Low-Dose Oxytocin } \\
(\mathrm{N}=\mathbf{7 2})\end{array}$ & $\begin{array}{l}\text { High-Dose Oxytocin } \\
(\mathrm{N}=70)\end{array}$ & $\operatorname{RR}(95 \% \mathrm{CI})$ \\
\hline \multicolumn{5}{|c|}{ Mild adverse effects, n (\%) } \\
\hline Nausea/Vomiting & $4(2.8)$ & $3(4.2)$ & I (I.4) & $2.27(0.34,14.94)$ \\
\hline Flushing & $17(12.0)$ & $7(9.7)$ & $10(14.3)$ & $0.69(0.29,1.67)$ \\
\hline Tachycardia & $13(9.2)$ & $5(6.9)$ & $8(11.4)$ & $0.63(0.23,1.75)$ \\
\hline \multicolumn{5}{|c|}{ Severe adverse effects, $n$ (\%) } \\
\hline Hypotension & $55(38.7)$ & $22(30.6)$ & $30(42.9)$ & $0.72(0.46, \mathrm{I} . \mathrm{II})$ \\
\hline Chest pain & $4(2.8)$ & I (I.4) & $3(4.3)$ & $0.42(0.06,2.74)$ \\
\hline ECG change & $2(1.4)$ & I (I.4) & $\mathrm{I}(\mathrm{I} .4)$ & $0.97(0.10,9.13)$ \\
\hline
\end{tabular}

Note: Data are presented as number (percentage).

Abbreviations: RR, relative risk; $\mathrm{Cl}$, confidence interval; $\mathrm{NICU}$, neonatal intensive care unit; $\mathrm{ECG}$, electrocardiographic.

contraction with a lower rate of methylergonovine maleate requirement. The first is the fact that oxytocin stimulates uterine contractility by binding to the myometrial oxytocin receptor. Higher concentrations of estrogen during pregnancy increase the density and kinetic binding of the oxytocin receptor and enhance uterine sensitivity to oxytocin. ${ }^{19}$ The second is that synthetic oxytocin as an intravenous bolus injection gives rise to peaks of oxytocin that are equivalent in size to peaks that occur during physiological birth and stimulate uterine contractions. A previous study of the plasma oxytocin levels in physiological labor and birth found that oxytocin levels doubled in response to doubling of the rate of infusion of synthetic oxytocin and administration of 4 to $8 \mathrm{IU}$ of synthetic oxytocin. ${ }^{20}$ The third possible explanation is the oxytocin receptor desensitization phenomenon. A previous in vitro study of the contractile response of the human term pregnant myometrium to oxytocin with different concentrations of oxytocin for variable duration observed an attenuated dose-response effect to increasing 
concentrations of oxytocin in terms of both amplitude and frequency of contractions. $^{21}$

Administration of oxytocin can result in nausea, vomiting, headache, and flushing. In large doses, it can lead to water retention, hyponatremia, seizure, and coma. ${ }^{10}$ The incidence of these side effects was low in our study, and did not differ significantly between groups. Electrocardiographic changes suggestive of myocardial ischemia may also occur as a result of oxytocin effects. A study by Pinder et al of the hemodynamic changes induced by rapid bolus of 5 or 10 units of oxytocin in 34 healthy term patients undergoing cesarean section found a small but statistically significant reduction in mean arterial pressure from baseline at $30 \mathrm{~s}$ after administration of the 10 -unit bolus. ${ }^{22}$ However, it also led to a large, statistically significant increase in heart rate and cardiac output. However, we cannot directly compare these results with those of our study due to the fact that Pinder et al measured change from the baseline, while we determined the number of women with clinically significant hypotension and/or tachycardia.

Jonsson et al studied electrocardiogram change suggestive of myocardial ischemia in patients administered 5 IU and $10 \mathrm{IU}$ of oxytocin $^{23}$ and found a significant difference in ST depression associated with oxytocin administration ( $7.7 \%$ and $21.6 \%$, respectively). By contrast, in our study, there were only of premature ventricular complex, no electrocardiogram changes suggestive of myocardial ischemia. This may be due to the fact that we monitored the electrocardiogram in real time rather than using a recording, while Jonsson et al used a Holter monitor to detect abnormalities.

\section{Conclusion}

A 5 IU bolus of oxytocin was noninferior to the standard 10 IU bolus for initiating adequate uterine contraction, required fewer additional uterotonic agents, and led to fewer oxytocin-related adverse events. A lower dosage of oxytocin might be considered in pregnant women undergoing caesarean section. Further studies should be conducted to evaluate the effectiveness of an oxytocin dosage of 3 IU or less or split-dose regimens in order to minimize oxytocin-related adverse events.

\section{Ethical Approval}

Ethical approval was obtained from the Khon Kaen University Ethics Committee for Human Research based on the
Declaration of Helsinki and the ICH Good clinical Practice Guidelines (September 25, 2018; Reference No. HE611369).

\section{Acknowledgment}

We would like to thank Dylan Southard, the English Consultant at Khon Kaen University's Faculty of Medicine, for his help in editing the English-language presentation of this manuscript.

\section{Funding}

This study was financially supported by the Khon Kaen University Faculty of Medicine (Thailand).

\section{Disclosure}

The authors have no conflicts of interest to report for this work.

\section{References}

1. The International Federation of Gynecology and Obstetrics. PPH leading to unnecessary deaths. 2018; Available from: https://www. figo.org/news/pph-leading-unnecessary-deaths-0016005.

2. Nathan LM. An overview of obstetric hemorrhage. Semin Perinatol. 2019;43(1):2-4. doi:10.1053/j.semperi.2018.11.001

3. Grobman WA, Bailit JL, Rice MM, et al. Frequency of and factors associated with severe maternal morbidity. Obstet Gynecol. 2014;123 (4):804-810. doi:10.1097/AOG.0000000000000173

4. Callaghan WM, Mackay AP, Berg CJ. Identification of severe maternal morbidity during delivery hospitalizations, United States, 1991-2003. Am J Obstet Gynecol. 2008;199(2):133.e1-8. doi:10. 1016/j.ajog.2007.12.020

5. Creanga AA, Berg CJ, Ko JY, et al. Maternal mortality and morbidity in the United States: where are we now? Journal of Women's Health. 2014;23(1):3-9. doi:10.1089/jwh.2013.4617

6. Committee on Practice Bulletins-Obstetrics. Practice Bulletin No. Practice bulletin no. 183: postpartum hemorrhage.Obstet Gynecol.2017;130(4):e168-86. doi:10.1097/AOG.0000000000002 351

7. Xu C, Fu Q, Tao H-B, et al. Effect of cesarean section on the severity of postpartum hemorrhage in Chinese women: the Shanxi study. Curr Med Sci. 2018;38(4):618-625. doi:10.1007/s11596-018-1922-1

8. Ononge S, Mirembe F, Wandabwa J, Campbell OMR. Incidence and risk factors for postpartum hemorrhage in Uganda. Reprod Health. 2016;13(1):38. doi:10.1186/s12978-016-0154-8

9. Dept of Reproductive Health and research, World Health Organization (WHO). WHO Recommendations for the Prevention and Treatment of Postpartum Haemorrhage. Geneva: WHO; 2012.

10. Bergum D, Lonnée H, Hakli TF. Oxytocin infusion: acute hyponatraemia, seizures and coma. Acta Anaesthesiol Scand. 2009;53 (6):826-827. doi:10.1111/j.1399-6576.2009.01964.x

11. Prevention and management of postpartum haemorrhage: green-top guideline No. 52. BJOG. 2017;124:pp. e106-e149.

12. Sentilhes L, Vayssière C, Deneux-Tharaux C, et al. Postpartum hemorrhage: guidelines for clinical practice from the French College of Gynaecologists and Obstetricians (CNGOF): in collaboration with the French Society of Anesthesiology and Intensive Care (SFAR). Eur J Obstet Gynecol Reprod Biol. 2016;198:12-21. doi:10.1016/j.ejogrb.2015.12.012 
13. Moher D, Hopewell S, Schulz KF, et al. CONSORT 2010 explanation and elaboration: updated guidelines for reporting parallel group randomised trials. BMJ. 2010;23(340):c869. doi:10.1136/bmj.c869

14. AHFS Drug Information 2004. In: McEvoy GK, editor. Oxytocin. Bethesda, MD: American Society of Health-System Pharmacists; 2004:3121-3123.

15. Holleboom CG, van Eyck J, Koenen SV, et al. Carbetocin in comparison with oxytocin in several dosing regimens for the prevention of uterine atony after elective caesarean section in the Netherlands. Arch Gynecol Obstet. 2013;287(6):1111-1117. doi:10.1007/s00404-0122693-8

16. Sarna MC, Soni AK, Gomez M, Oriol NE. Intravenous oxytocin in patients undergoing elective cesarean section. Anesth Analg. 1997;84 (4):753-756. doi:10.1213/00000539-199704000-00010

17. Butwick AJ, Coleman L, Cohen SE, Riley ET, Carvalho B. Minimum effective bolus dose of oxytocin during elective Caesarean delivery. Br J Anaesth. 2010;104(3):338-343. doi:10.1093/bja/aeq004

18. Derbel M, Mekki D, Riahi A, Dimassi K, Mebazaa MS, Gara MF. Comparison of two oxytocin administration protocols in elective cesarean section: 5 iu versus 10 iu. Tunis Med. 2016;94(4):253-258.

19. Fuchs AR, Ivell R, Ganz N, Fields MJ, Gimenez T. Secretion of oxytocin in pregnant and parturient cows: corpus luteum may contribute to plasma oxytocin at term. Biol Reprod. 2001;65 (4):1135-1141. doi:10.1095/biolreprod65.4.1135
20. Uvnäs-Moberg K, Ekström-Bergström A, Berg M, et al. Maternal plasma levels of oxytocin during physiological childbirth a systematic review with implications for uterine contractions and central actions of oxytocin. BMC Pregnancy Childbirth. 2019;19 (1):285. doi:10.1186/s12884-019-2365-9

21. Balki M, Erik-Soussi M, Kingdom J, Carvalho JCA. Oxytocin pretreatment attenuates oxytocin-induced contractions in human myometrium in vitro. Anesthesiology. 2013;119(3):552-561. doi:10.1097/ ALN.0b013e318297d347

22. Pinder AJ, Dresner M, Calow C, Shorten GD, O'Riordan J, Johnson R. Haemodynamic changes caused by oxytocin during caesarean section under spinal anaesthesia. Int J Obstet Anesth. 2002;11 (3):156-159. doi:10.1054/ijoa.2002.0970

23. Jonsson M, Hanson U, Lidell C, Nordén-Lindeberg S. ST depression at caesarean section and the relation to oxytocin dose. BJOG. 2010;117(1):76-83. doi:10.1111/j.1471-0528.2009.02356.x

\section{Publish your work in this journal}

The International Journal of Women's Health is an international, peerreviewed open-access journal publishing original research, reports, editorials, reviews and commentaries on all aspects of women's healthcare including gynecology, obstetrics, and breast cancer. The manuscript management system is completely online and includes a very quick and fair peer-review system, which is all easy to use. Visit http://www.dovepress.com/testimonials.php to read real quotes from published authors. 